vasCuLAR DISEASE

\title{
A new organoid model to study diabetic vasculopathy
}

Patients with diabetes mellitus are at high risk of developing severe complications caused by blood vessel dysfunction, such as blindness, kidney failure, limb amputations or cardiovascular disease. The mechanisms underlying diabetic vasculopathy are still not fully understood, which limits therapeutic drug development. A new study published in Nature reports the generation of human blood vessel organoids as a new model to investigate diabetes-related vascular changes and identify future treatments.

"Inspired by previous work from the labs of Christine Mummery and Sharon Gerecht, we had the ambitious goal to generate fully functional blood vessels from human induced pluripotent stem cells (iPSCs) to model diabetic vasculopathy," explains lead investigator, Josef Penninger.

Vascular organoids were generated from human iPSCs using a multistep protocol that included in vitro differentiation of the cells towards the mesoderm lineage, vascular specification

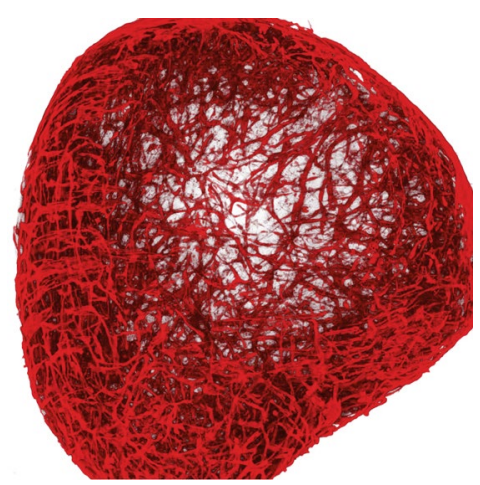

$3 \mathrm{D}$ reconstruction of capillary organization in a vascular organoid. Reproduced from Wimmer, R. A. et al. Human blood vessel organoids as a model of diabetic vasculopathy. Nature 565, 505-510 (2019) and embedment into a 3D matrix. Analysis of the organoids revealed well-organized blood vessels composed of endothelial cells, pericytes and an associated basement membrane. Gene expression profiling confirmed that endothelial cells had a typical endothelial gene signature and expressed maturity markers, such as von Willebrand factor.

Previous studies published by Rakesh Jain have shown that the co-implantation of human endothelial cells and mesenchymal cells in mice generates stable, functional blood vessels. These results prompted the investigators to examine the vasculogenic potential of the organoids in vivo. Transplantation of organoids into mice revealed that human blood vessels could survive in vivo and functionally connect to the mouse vasculature. "We were able to show that we can grow a whole vascular tree in the mouse, including arteries, arterioles, capillaries and venules, that is entirely human and stays engrafted for more than 6 months," says Reiner Wimmer, who contributed to the study.

Next, the investigators cultured the organoids in an increased concentration of glucose to mimic diabetic conditions. Hyperglycaemia induced thickening of the human vessels with induction of the expression of type IV collagen and of other basement membrane components, such as fibronectin. "Basement membrane thickening is a very poorly understood process but is believed to contribute to vascular dysfunction in patients with diabetes," explains Wimmer. Similar thickening of the basement membrane was observed in human blood vessels grown in streptozotocin-induced diabetic mice, providing further validation of the organoid model to study diabetic vascular complications. Interestingly, iPSC-derived endothelial cells alone exposed to diabetic medium did not show an increase in type IV collagen levels, suggesting that pericytes contribute to the changes observed in the vascular basement membrane.

Drugs commonly used for the treatment of diabetes were inefficient at preventing the thickening of the basement membrane induced by the diabetic medium. By contrast, DAPT, a $\gamma$-secretase inhibitor that blocks the NOTCH pathway, abrogated the thickening of the basement membrane in the organoids in vitro and in the diabetic mouse. Blockade of delta-like protein 4 (DLL4) and NOTCH3, two components of the NOTCH pathway, also blocked the increase in type IV collagen production induced by hyperglycaemia in vitro.

"With our model we found that an intricate endothelial-pericyte interaction via the NOTCH3 receptor (expressed on pericytes) and the ligand DLL4 (expressed on the endothelial cell) are key mediators of diabetes-mediated vascular basement membrane thickening," says Penninger. The investigators plan to use the new organoid model to identify drugs that could block NOTCH3 and DLL4 without causing adverse effects and to study other vascular diseases. "We also envision developing a bank of donor iPSCs that we can then readily differentiate into vascular organoids for transplantation into patients with severe burns, non-healing wounds in diabetes or for in vivo revascularization of damaged tissues such as the heart," concludes Penninger.

Alexandra Le Bras

ORIGINAL ARTICLE Wimmer, R. A. et al. Human blood vessel organoids as a model of diabetic vasculopathy. Nature 565, 505-510 (2019) 